\title{
Evaluación de carbono total en bosque de tara (Caesalpinia spinosa Molina Kuntze): Centro Poblado
} Señor de los Milagros

\section{Total carbon assessment in the Tara forest (Caesalpinia spinosa Molina Kuntze): Señor de los Milagros Populated Center}

\author{
Melissa Lucerito Epiquin Rojas ${ }^{1 *}$, Alcides Román Peña ${ }^{1}$, Elder Chichipe Vela ${ }^{1}$, Marielita Arce Inga ${ }^{1}$
}

\section{RESUMEN}

El objetivo fue evaluar el carbono total en bosque de tara en suelo, biomasa aérea y hojarasca, en el anexo Señor de los Milagros, distrito de Magdalena (Departamento Amazonas). Para realizar esta investigación se utilizaron 15 unidades muestrales. En la determinación de carbono en suelo se tomaron dos muestras del pie de cada unidad muestral, de $0-0,15 \mathrm{~m}$ y de $0,15-0,30 \mathrm{~m}$. Las muestras fueron llevadas al laboratorio para su análisis. En la determinación de carbono en biomasa aérea y hojarasca se realizó la medición de variables dasométricas antes del derribo y se tomaron muestras de hojas, ramas yfuste, para ser pesados en campo y secados en laboratorio a $75^{\circ} \mathrm{C}$. Los resultados obtenidos en el componente suelo fueron de $15,49 \mathrm{tnC} / \mathrm{ha}$, y biomasa aérea de $64,39 \mathrm{tnC} / \mathrm{ha}$, equivalente a $56,81 \mathrm{tnCO}_{2} /$ ha y $236,12 \mathrm{tnCO}_{2} /$ ha, respectivamente. En la determinación de ecuaciones alométricas, se obtuvo que el mejor predictor de biomasa es el DAP, generando relaciones alométricas con $\mathrm{R}^{2}$ mayor a 0.873 . La ecuación que mejor se ajusta para estimar el contenido de biomasa seca en tara es la ecuación cuadrática $\mathrm{Bt}_{(\mathrm{kgg})=}$ $190,982+61,743^{*}$ DAP-1,991*DAP ${ }^{2}$. Finalmente se calculó que los bosques de tara secuestran un aproximado de 34 565,74tnCO2/118ha en el distrito de Magdalena, que en términos económicos equivale a US\$ 446 243,70. Esto demuestra que tienen un gran potencial para el mercado de carbono, lo cual sería una fuente de ingresos futura en caso de que se establezcan negociaciones o créditos de carbono.

Palabras claves: Caesalpinia spinosa, carbono, biomasa seca, hojarasca, ecuación alométrica.

\begin{abstract}
The goal was to evaluate the total carbon in tara forest, both in soil, aerial biomass and leaf litter, in the Señor de los Milagros Populated Center, Magdalena district. To carry out this research, 15 sample units were used; in the determination of carbon in soil, 2 samples were taken from the foot of each sample unit, $0-0.15 \mathrm{~m}$ and $0.15-0.30 \mathrm{~m}$; samples were taken to the laboratory for analysis. In the determination of carbon in aerial biomass and leaf litter, the measurement of dasometric variables was performed before felling and samples were taken from leaves, branches, stem, to be weighed in the field and dried in the laboratory at $75^{\circ} \mathrm{C}$. The results obtained in the soil component were $15.49 \mathrm{tnC} / \mathrm{ha}$ and aerial biomass of $64.39 \mathrm{tnC} / \mathrm{ha}$, equivalent to $56.81 \mathrm{tnCO} /$ / ha and $236.12 \mathrm{tnCO} 2 /$ ha respectively. In the determination of allometric equations, it was obtained that the best predictor of biomass is the DAP, generating allometric ratios with $\mathrm{R}^{2}$ greater than 0.873 . The equation that best fits to estimate the dry biomass content in tara is the quadratic equation $\mathrm{Bt}_{(\mathrm{kg})}=-190.982+61.743 * \mathrm{DAP}-1.991 * \mathrm{DAP}^{2}$. Finally, it was calculated that the tara forests sequester an approximate of $34565.74 \mathrm{tnCO} / 118$ ha in the district of Magdalena, which in economic terms equals US\$ 446243,70 , demonstrating that they have great potential for the carbon market, which would be a source of income future in case negotiations or carbon credits are established.
\end{abstract}

Keywords: Caesalpinia spinosa, carbon, dry biomass, litter, allometric equation.

\footnotetext{
${ }^{1}$ Universidad Nacional Toribio Rodríguez de Mendoza de Amazonas (UNTRM-A), Instituto de Investigación para el Desarrollo Sustentable de Ceja de Selva, Calle Higos Urco N³42-350-356, Calle Universitaria N³04, Chachapoyas, Perú

"Autor de correspondencia. E-mail:mepquin@indes-ces.edu.pe
} 


\section{INTRODUCCIÓN}

La concentración de gases de efecto invernadero (GEI) va en aumento, incrementando el calentamiento de la tierra y causando preocupación a nivel mundial. Uno de los principales responsables de este efecto invernadero es el $\mathrm{CO}_{2}$ en la atmósfera, causado por las actividades antropogénicas, afectando el equilibrio de radiación del planeta (IPCC, 2001). Razón por la cual se deben tomar medidas para reducir las emisiones de este gas e incrementar su captura en los suelos y biomasa, adoptando nuevas tecnologías y políticas apropiadas en el manejo de bosques y agricultura (FAO, 2002). Los sistemas forestales y agroforestales son algunos de los grandes sumideros de $\mathrm{CO}_{2}$, ya que, con las especies y manejo adecuado, contribuyen al secuestro de carbono atmosférico, recuperando simultáneamente áreas degradadas (Douterlungne et al., 2013).

El Departamento Amazonas posee grandes bosques donde se pueden localizar diversas especies arbóreas, entre ellas la tara. Los árboles de tara son plantas nativas que se adaptan a cualquier tipo de terreno teniendo por ende una tasa alta de supervivencia y crecimiento en áreas degradadas. A nivel internacional no son muy numerosas las investigaciones relacionadas con captura de carbono en árboles de tara, sin embargo encontramos investigaciones como la de Díaz-Gustavo et al. (2015), en la cual evaluó la Caesalpinia platyloba como una alternativa para la retención de carbono atmosférico y como una actividad económica factible y viable en los mercados de carbono. Pérez et al. (2015), en su investigación con Caesalpinia velutina, reportaron un alto contenido de carbono y por consiguiente un alto almacenamiento de carbono a escala de calle y casco urbano. A nivel nacional encontramoQuispe et al. (2012), los cuales determinaron el almacenamiento de carbono atmosférico de tara, cuantificando la producción de biomasa y el contenido de carbono en el tallo, ramas y follaje. A nivel local encontramos estudios como el de Pérez y Oliva (2013), que en su estudio determinaron el carbono en la biomasa aérea de un sistema agroforestal de produc- ción de café, en asociación con morocho, tara, guaba y huarango.

En el centro poblado Señor de los Milagros, distrito de Magdalena, se ha venido deforestando gran parte de estos bosques naturales de tara, siendo utilizados como fuente de energía. En la actualidad estos parches ecológicos están siendo reforestados con la misma especie por los grandes beneficios económicos que les generan los frutos a la población. En base a lo mencionado, mediante esta investigación se busca determinar cuan grande es la capacidad de captura de carbono total del suelo y biomasa aérea de la rara, para que de esta manera se conozca el potencial de esta especie como una alternativa a obtener fondos para conservar estos bosques.

\section{MATERIALY MÉTODOS}

\section{Ubicación del área de estudio}

La investigación se realizó en el Centro Poblado Señor de los Milagros del distrito de Magdalena; provincia de Chachapoyas, Departamento Amazonas (Figura 1). Este caserío se encuentra a orillas del río Utcubamba y abarca una superficie de $135,47 \mathrm{~km}^{2}$ ubicándose a una altitud media de $1980 \mathrm{~m} \mathrm{s.n.m.}$

\section{Población, muestra y muestreo}

La población estuvo constituida por todas las áreas con árboles de tara del Centro Poblado Señor de los Milagros. Se utilizó un muestreo no probabilístico, en el cual, la selección de las muestras fue de tres parcelas. En cada parcela se seleccionaron cinco unidades muestrales de tara al azar. Para la toma de muestras en hojarasca se utilizó el método del cuadrante y para suelo, se realizaron calicatas de $0.30 \mathrm{~m}$ de profundidad en cada una de las unidades muestrales.

Métodos, técnicas e instrumentos de recolección de datos

Se utilizó el método destructivo, ya que este método arroja resultados de alta confiabilidad. Para la recolección de datos se utilizaron fichas de colecta de información de muestras tomadas en campo y fichas de laboratorio. 


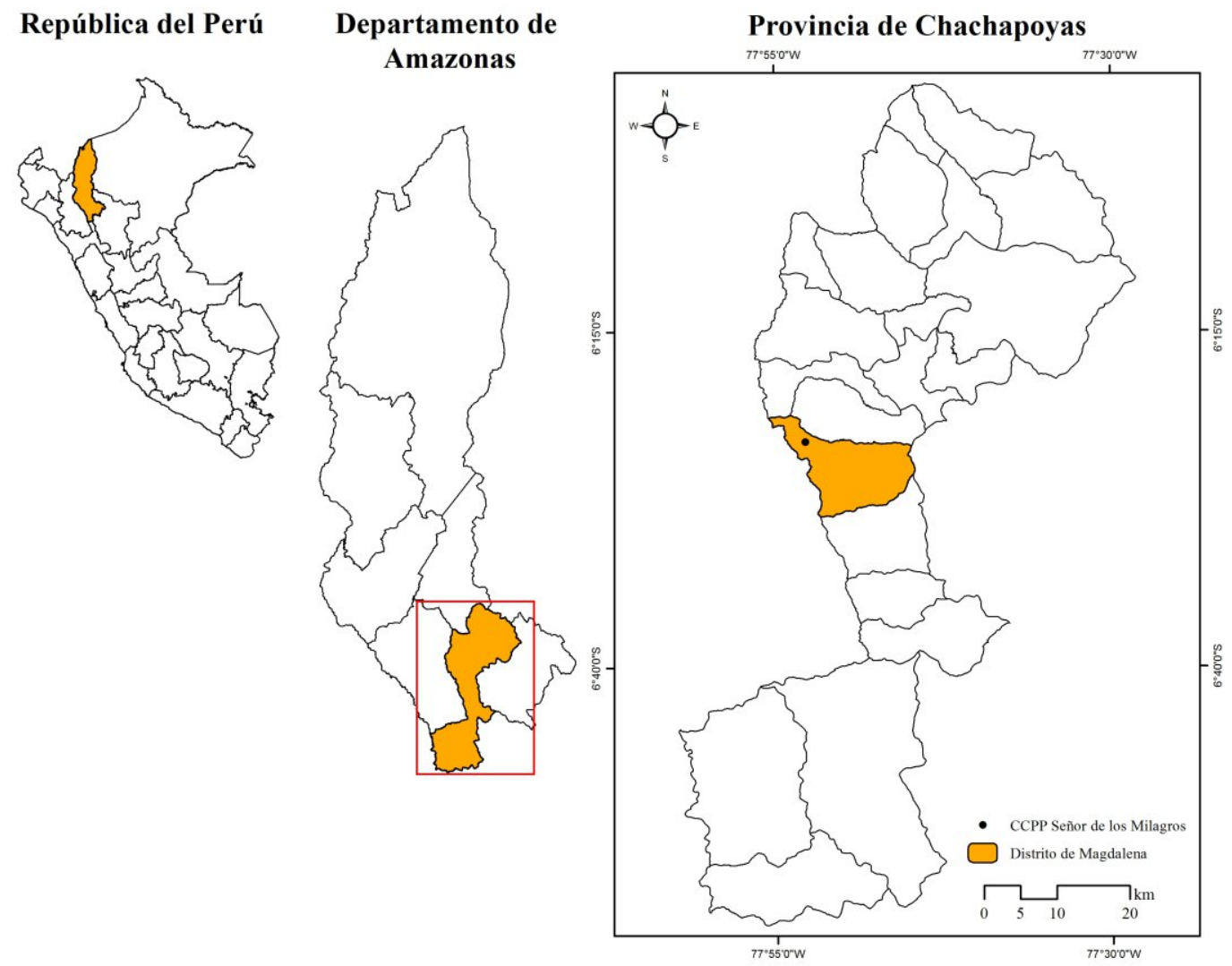

Figura 1. Ubicación geográfica del área de estudio en el Centro Poblado Señor de los Milagros del distrito de Magdalena (Provincia de Chachapoyas).

\section{Procedimiento}

\section{Fase de campo}

\section{1) Selección de parcelas experimentales}

Las parcelas seleccionadas tuvieron el mismo microclima, condiciones de uso de suelo, estado de conservación agronómica y perturbación de la vegetación.

\section{2) Caracterización de parcelas experimentales}

Se realizó la identificación y caracterización de parcelas experimentales mediante el sistema de Geoposicionamiento Global (GPS). Se midió la pendiente con la ayuda de un distanciómetro Leica Disto D8, y por último, se hizo una caracterización fisicoquímica del suelo.

3) Toma de muestras de suelo para análisis de materia orgánica

Se utilizó la metodología propuesta por el Reglamento para la ejecución de levantamiento de suelos, establecido mediante Decreto Supremo No 013-2010-AG (MINAGRI, 2010). Por ello se tomaron muestras al pie de cada una de las unidades muestrales, logrando de esta manera tener definidos los horizontes entre 0 $0,15 \mathrm{~m}$ y $0,15-0,30 \mathrm{~m}$.

\section{4) Toma de muestras de suelo para densidad}

Con un martillo de pico se clavó un cilindro muestreador hasta una profundidad de $10 \mathrm{~cm}$, y se extrajo la muestra con cuidado evitando pérdidas de suelo. Se retiró el exceso de suelo para que este quedase nivelado siguiendo los bordes del anillo del cilindro. Por último, se colocaron en una bolsa de polipropileno para ser pesados en campo y posteriormente llevados a laboratorio.

\section{5) Toma de muestras de biomasa aérea}

Se utilizó la metodología empleada por Picard et al. (2012). Para ello se extrajo la parte aérea de 5 unidades muestrales en cada una de las parcelas. Antes del derribo se midió el diámetro a la altura del pecho (DAP). Se midió la altura del fuste limpio, se seccionó el fuste y las ramas de tal forma que el fuste se seccionó en tamaños de $2 \mathrm{~m}$ de largo, del cual se extrajeron rodajas de 5 $\mathrm{cm}$ de espesor. Las ramas y el fuste fueron pesados en 
campo y se tomaron muestras para ser llevadas al laboratorio.

\section{6) Toma de muestras de hojarasca}

Se cuantificaron los materiales muertos procedentes de los árboles de tara como hojas, flores y frutos, en cuadrantes de $0,5 \mathrm{~m}$ x $0,5 \mathrm{~m}$, se pesaron en campo y se trasladaron al laboratorio

\section{Fase de laboratorio}

\section{1) Análisis de suelo}

Para el análisis de materia orgánica (M.O) del suelo se utilizó el método de Walkley y Black.

2) Análisis de muestras de biomasa y hojarasca Las muestras de ramas, fuste, hojas y hojarasca fueron secadas en la estufa a $75^{\circ} \mathrm{C}$ hasta obtener peso constante (González, 2008).

\section{Fase de gabinete}

Para la determinación de carbono orgánico de suelo y biomasa aérea se realizó lo siguiente:

1) Estimación del Carbono Orgánico en el Suelo (COS)

La estimación del COS se calculó por hectárea (Gorbitz, 2001):

$\mathrm{Ws}=\mathrm{a} \times \mathrm{p} \times \mathrm{da}$

Donde:

$\mathrm{Ws}=$ peso del suelo

$\mathrm{a}=$ área (ha)

$\mathrm{p}=$ profundidad

$\mathrm{d}=$ densidad aparente $\left(\mathrm{Kg} / \mathrm{m}^{3}\right)$.

Luego:

$\mathrm{CO}(\mathrm{tn} / \mathrm{ha})=\mathrm{Ws}_{\mathrm{s}} \times \mathrm{CO}$

Donde:

$\mathrm{CO}=$ carbono orgánico

$\mathrm{Ws}=$ peso del suelo calculado.

\section{2) Determinación de Biomasa Seca}

La biomasa seca se determinó mediante la relación peso seco - peso fresco de las muestras en cada componente (González, 2008):

$\mathrm{BSc}=(\mathrm{PS} / \mathrm{PH}) \times \mathrm{Bhc}$

Donde:

$\mathrm{BSc}=$ biomasa seca del componente $(\mathrm{kg})$

$\mathrm{PS}=$ peso seco de la muestra $(\mathrm{g})$

$\mathrm{PH}=$ peso húmedo de la muestra $(\mathrm{g})$

$\mathrm{BHc}=$ biomasa húmeda del componente $(\mathrm{kg})$.

La biomasa seca total de cada árbol es determinada sumando la biomasa seca de cada uno de sus Componentes (González, 2008):

$\mathrm{BS}$ total $=\mathrm{BS}$ fuste $+\mathrm{BS}$ ramas $+\mathrm{BS}$ hojas

Donde:

BS total $=$ Biomasa total

BS fuste $=$ Biomasa fuste

$\mathrm{BS}$ ramas $=$ Biomasa ramas

BS hojas $=$ Biomasa hojas

\section{3) Determinación de biomasa de hojarasca}

Se determinó por medio de la relación de peso fresco total, peso fresco de toda la muestra y el peso seco (Gorbitz, 2001).

$\mathrm{Bh}(\mathrm{tn} / \mathrm{ha})=((\mathrm{PSM} / \mathrm{PFM}) \times \mathrm{PFT}) \times 0,004$

Donde

$\mathrm{Bh}=$ biomasa de hojarasca

$\mathrm{PSM}=$ peso seco de la muestra colectada $(\mathrm{g})$

$\mathrm{PFM}=$ peso fresco de la muestra colectada $(\mathrm{g})$

$\mathrm{PFM}=$ peso fresco total por metro cuadrado $(\mathrm{g})$

$0,004=$ Factor de conversión 


\section{4) Cálculo de la Biomasa Vegetal Total}

Este se calcula sumando la biomasa arbórea y la biomasa de hojarasca (Gorbitz, 2001).

$\mathrm{BVT}(\mathrm{tn} / \mathrm{ha})=\mathrm{BA}+\mathrm{Bh}$

Donde:

BVT $=$ biomasa vegetal total $(\mathrm{t} / \mathrm{ha})$

$\mathrm{BA}=$ biomasa arbórea

$\mathrm{Bh}=$ biomasa de hojarasca.

\section{5) Determinación de Carbono Total}

Se realiza multiplicando la biomasa seca por el factor 0,5 , el factor promedio de carbono registrado en especies vegetales (Gorbitz, 2001).

$\mathrm{CBV}$ individuo $=\mathrm{BS}$ total individuo $\times 0,5$

Donde:

$\mathrm{CBV}=$ carbono total en la biomasa vegetal

$\mathrm{BS}=$ biomasa seca

$0,5=$ Factor de conversión.

El resultado del carbono total en la biomasa se multiplica por el factor de conversión de 3,666, para obtener la proyección de $\mathrm{CO}_{2}$ capturado por las plantaciones de tara (Valenzuela, 2001).

6) Formulación de ecuaciones alométricas método indirecto:

Se formularon ecuaciones, en base a variables dasométricas como altura de fuste, DAP y biomasa, para estimar las reservas de carbono en función de la masa seca de materia orgánica.

\section{7) Análisis de datos}

Los resultados se analizaron mediante la prueba $\mathrm{T}$ de Student al $5 \%$ de significancia para ver las diferencias significativas del contenido de $\mathrm{CO}_{2}$ en las diferentes profundidades del suelo.

Para la formulación de ecuaciones alométricas, se hizo un análisis estadístico consistente en pruebas de regre- sión múltiple, con el objetivo de definir la mejor correlación $\left(\mathrm{R}^{2}\right)$ entre la variable dependiente respecto a las independientes, utilizando el software estadístico SPSS v. 20.0

\section{RESULTADOS}

Se identificaron tres parcelas con árboles de tara, las cuales se encontraban en un valle interandino, con un relieve accidentado y montañoso, a una altura aproximada de $1827 \mathrm{~m}$

s. n. m., y una pendiente de $36^{\circ}$. Estas parcelas son partes de bosques naturales pero se encuentran conservados por los mismos dueños, dándoles un manejo agronómico de deshierbas ypodas continuas. Los análisis de caracterización de suelo a diferentes profundidades arrojaron que son suelos franco arcillo arenosos, moderadamente alcalinos, con una fertilidad media, con alta materia orgánica y una densidad de $0.99 \mathrm{~g} / \mathrm{m}^{3}$, aproximadamente.

\section{Cálculo de almacenamiento de carbono en el suelo}

El resultado de COS fue de 15,49 tnC/ha aproximadamente, equivalente a $56,81 \mathrm{tnCO}_{2} / \mathrm{ha}$. Como se observa en la Tabla 1 en las muestras de $0-0,15 \mathrm{~m}$ el carbono orgánico es mayor a las de $0,15-0,30 \mathrm{~m}$, obteniendo valores promedios de 7,99 $\mathrm{tnC} / \mathrm{ha}$ y 7,50 tnC/ha, respectivamente. Estos valores de acuerdo a la prueba $\mathrm{T}$ de Student al 5\% de significación, no mostraron diferencias significativas con respecto a la profundidad de muestreo.

Tabla 1. Carbono orgánico en el suelo (COS)

\begin{tabular}{ccc}
\hline $\mathbf{N}^{\circ}$ Parcela & Muestra $(\mathbf{m})$ & $\mathbf{C O S}(\mathbf{t n C} / \mathbf{h a})$ \\
\hline \multirow{2}{*}{1} & $0-0,15$ & 7,35 \\
\cline { 2 - 3 } & $0,15-0,30$ & 6,00 \\
\cline { 2 - 3 } 2 & $0-0,15$ & 9,52 \\
\cline { 2 - 3 } 3 & $0,15-0,30$ & 9,29 \\
\cline { 2 - 3 } 3 & $0-0,15$ & 7,11 \\
\hline
\end{tabular}

\section{Calculo de carbono total}

\section{Biomasa seca (Bs)}

El resultado obtenido fue que los árboles de tara tienen un aproximado de $115,93 \mathrm{Kg}$ de biomasa seca por árbol, equivalente a 128,79 t/ha. En la figura 2 se muestra el porcentaje de biomasa de cada uno de los componentes de la tara, en el cual se observa que el 
almacenamiento de carbono se encuentra distribuido en mayor cantidad en el fuste $(69,87 \%)$, seguido de las ramas $(24,02 \%)$ y por último en las hojas $(6,10 \%)$.

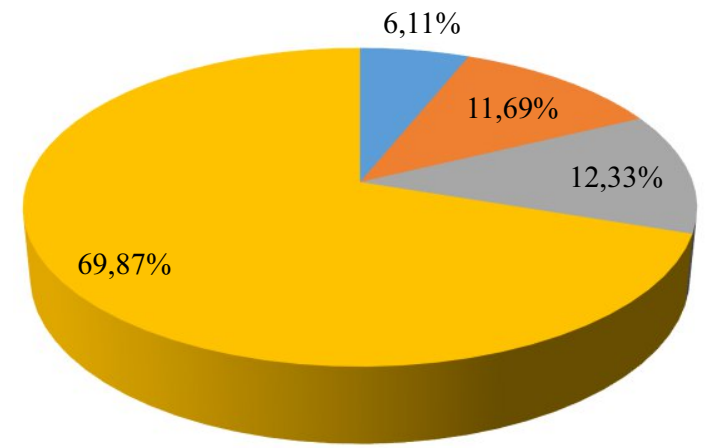

- Hojas $\square$ Ramas secundarias $\square$ Ramasterciarias $\square$ Fuste

Figura 2. Porcentaje de biomasa aérea de cada uno de los componentes de la tara.

\section{Biomasa de hojarasca $(B h)$}

Se obtuvo que un árbol de tara tiene $0,0832 \mathrm{~g}$ de biomasa de hojarasca, el cual equivale a $9,24 \times 10^{-5} \mathrm{t} / \mathrm{ha}$.

\section{Carbonototal (CT)}

El resultado obtenido fue que los árbol de tara tiene $57,96 \mathrm{Kg}$ de carbono en su biomasa vegetal, equivalente a 64,39 tnC/ha. La proyección de $\mathrm{CO}_{2}$ fue de $212,53 \mathrm{Kg}$ de $\mathrm{CO}_{2}$ por árbol aproximadamente, haciendo un promedio de 236,12 $\mathrm{tnCO}_{2} /$ ha, que los árboles de tara capturan. La cantidad aproximada de carbono que secuestra el bosque de tara es de 34 565,74 tnCO2 / 118 ha del distrito de Magdalena, que en términos económicos equivale a US\$ 446 243,70.

\section{Ecuación alométrica}

Las ecuaciones se construyeron en base a 15 unidades muestrales de tara. Para realizar la construcción de las ecuaciones alométricas se hizo una regresión con una sola variable, utilizando la variable DAP, con la cual se pudo llegar a la conclusión que, la ecuación cuadrática $\mathrm{Bt}_{(\mathrm{kg})}=-190,982+61,743 \times \mathrm{DAP}-1,991 \times \mathrm{DAP}^{2}$, es la ecuación que más se ajusta para estimar la cantidad de carbono y $\mathrm{CO}^{2}$ por árbol de tara en una determinada área en el distrito de Magdalena, presentando un coeficiente de determinación del 0,873 , lo que significa que el DAP expresa la cantidad de carbono en un $87 \%$, cuyo valor tiene un nivel de confianza aceptable. Estos datos se ven reflejados en la Figura 3, donde a mayor DAP la biomasa va en aumento en la curva de la ecuación.

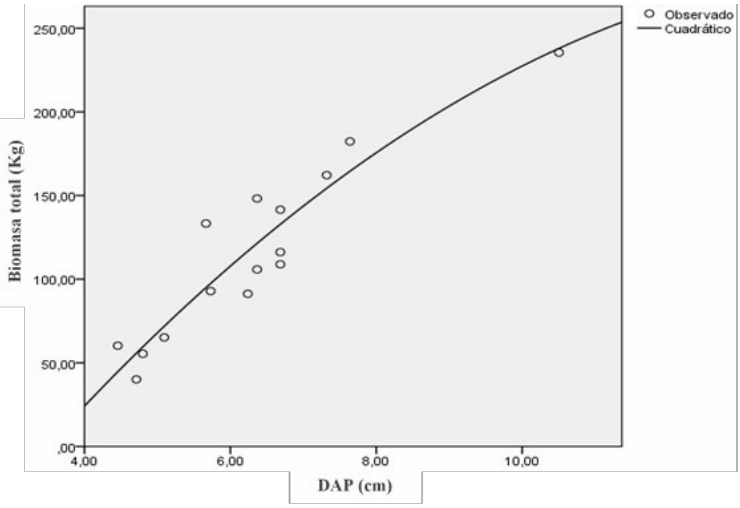

Figura 3. Relación entre DAP y BS

\section{IV.DISCUSIÓN}

Los análisis de caracterización de suelo, proyectan resultados de una textura franco arcillo arenoso predominante, un $\mathrm{pH}$ moderadamente alcalino, con una fertilidad media y un porcentaje alto de materia orgánica. Estos son algunos de los factores que influyen en la descomposición de la materia orgánica y así en las reservas de carbono en el componente suelo. Las reservas de carbono orgánico en un suelo están íntimamente relacionadas con el contenido de materiales finos del mismo, ya que, estas preservan la materia orgánica (Romanya et al., 2007). Además, cuanto mayor sea el contenido de carbono orgánico del suelo, mayor será el tamaño de los agregados, existiendo relación entre ambos (Martínez et al, 2008). Por otro lado el pH da las condiciones para aumentar los parámetros de carbono y nitrógeno, y por ende también el porcentaje de materia orgánica (Luza, 2015). La materia orgánica es capaz de aumentar la

capacidad tampón del suelo, debido a los grupos activos presentes (Martínez et al, 2008).

Los resultados de los análisis de $\mathrm{C}$ y $\mathrm{N}$ en el suelo son mayores en las muestras de 0 a 0,15 m. Esta tendencia tiene bastante similitud con la de Luza (2015), quien menciona que la mayor cantidad de $\mathrm{C}$ y $\mathrm{N}$ en el suelo se encuentra entre los $0-10 \mathrm{~cm}$. Sin embargo, estos componentes en suelo pueden variar dependiendo del tipo de uso del suelo, la cobertura vegetal y prácticas de manejo (Carvajal et al., 2009).

Los valores de densidad aparente estuvieron entre 0,89 y $1,06 \mathrm{~g} / \mathrm{m}^{3}$, que corresponden a valores bajos $\mathrm{y}$, por la 
relación directamente proporcional que existe entre la densidad aparente y la compactación, se puede decir que se trata de suelos no compactados (Blanco, 2009). Esta condición favorable de suelo se debe a las cantidades altas de materia orgánica, ya que ésta mejora propiedades como la porosidad del suelo, evitando problemas como la compactación (Martínez et al., 2008).

Los valores de materia orgánica encontrados en el suelo van desde 4,53 a $6,07 \%$, lo que indica que se tiene un suelo de buena calidad, ya que la materia orgánica del suelo es un indicador clave, tanto para temas de productividad como para la provisión de servicios ambientales (García et al., 2012). Además, los valores encontrados de materia orgánica superan el 4\% necesario para ser considerados como suelos orgánicos (Vela et al., 2011).

El promedio de carbono orgánico en suelo para la capa superficial $(0-0,15 \mathrm{~m})$ fue de 7,99 tnC/ha, y para la capa de $0,15-0,30 \mathrm{~m}$ fue de 7,50 tnC/ha. Estos resultados coinciden con Carvajal et al. (2009), quienes mencionan que la profundidad influye en el contenido de materia orgánica. Sin embargo, los depósitos de carbono almacenadosson mayores y varían en los primeros $30 \mathrm{~cm}$ del suelo, por lo que se presume que este va disminuyendo conforme aumenta la profundidad del mismo y se prefiere evaluar solo en este espesor (Cifuentes et al., 2004). Estos resultados concuerdan con Zambrano et al. (2004), quienes mencionan que existe una mayor concentración de carbono cerca de la superficie del suelo, es decir, en los macroagregados, proveniente de la biomasa microbiana y el carbono mineralizable. La cantidad de carbono capturado por las plantaciones de tara de esta investigación fue de 64,39 tnC/ha, el cual equivale a 236,12 tnCO $/$ ha. Estos resultados se pueden comparar con los obtenidos por Quispe et al. (2012), quienes obtuvieron un valor de 0,1131 tnC/ha en plantaciones de Caesalpinia spinosa de 8 meses de edad. También existen investigaciones con otras especies que pertenecen a la misma familia, como la de Díaz-Gustavo et al. (2015), quienes evaluaron plantaciones de Caesalpinia platyloba, repor- tando un contenido de carbono promedio de 99,15 tnC/ha. También, Pérez et al. (2015), reportaron en su investigación un valor de 423,8 KgC/árbol en Caesalpinia velutina. Siendo de esta manera una buena opción para almacenamiento de carbono atmosférico a largo plazo por sus tasas altas de supervivencia y crecimiento rápido (Kanowski y Catterall, 2010).

Las ecuaciones con términos cuadráticos o cúbicos son útiles (Chave et al., 2005) y reflejan cambios en las tasas de acumulación de biomasa, probablemente debido a la proporción cambiante de tejidos lignificados y blandos durante los estados juveniles y maduros (Douterlungne et al., 2013). En el caso de la ecuación cuadrática resultante para esta investigación, se tiene como variable regresora al DAP, que es una variable más fácil de medir en comparación a la altura total del árbol. La validez de la ecuación alométrica está fundamentada en el hecho de que existe una relación proporcional entre los crecimientos relativos de los componentes de un individuo (Picard et al., 2012).

\section{CONCLUSIONES}

La caracterización de las áreas con árboles de tara permitió la evaluación de parámetros fisicoquímicos del suelo. Como resultado se obtuvo un $\mathrm{pH}$ alcalino, una conductividad eléctrica de 0,34, 13,96 ppm de $\mathrm{P}$, 306,69 ppm de K, 3\% de C, 5, 18\% M.O, 0,26\% de N, y una clase textural franco arcillo arenosa.

La cantidad de carbono almacenado en suelo fue de $15,49 \mathrm{tnC} / \mathrm{ha}$, equivalente a $56,81 \mathrm{tnCO}_{2} / \mathrm{ha}$, y en la

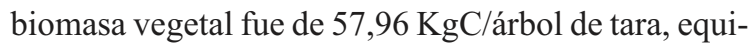
valente a $212,53 \mathrm{KgCO}_{2}$ /árbol.

Se evaluó el almacenamiento de carbono por hectárea sembrada con árboles de tara dándonos como resultado 64,39 tnC/ha, equivalente a 236,12 tnCO $/$ ha.

En cuanto a la ecuación alométrica seleccionada para estimar el contenido de biomasa total en árboles de tara, que posteriormente describirá el comportamiento del carbono en el distrito de Magdalena, está definida por la ecuación cuadrática.

La cantidad secuestrada de carbono del bosque de tara es de 34 565,74tnCO $\mathrm{CO}_{2} / 118 \mathrm{ha}$ del distrito de Magdalena 
aproximadamente, equivalente a US\$446 243,70, demostrando de esta manera que las plantaciones de tara tienen un gran potencial de almacenamiento y captura de carbono.

\section{REFERENCIAS BIBLIOGRÁFICAS}

Blanco, R. 2009. “La Relación Entre La Densidad Aparente Y La Resistencia Mecánica Como Indicadores De La Compactación Del Suelo.” Agrociencia 43(3): 231-239.

Carvajal A. F., A. Feijoo, H. Quintero, M. A. Rondón. 2009. “Carbono Orgánico Del Suelo En Diferentes Usos Del Terreno De Paisajes Andinos Colombianos." Revista de la Ciencia del Suelo y Nutricon Vegetal 9(3): 222-235.

Chave, J., C. Andalo, S. Brown, y M. A. Cairns. 2005. "Tree Allometric and Improved Estimation of Carbono Stocks and Balance in Tropical Forests." Oecologia 145: 87-99.

Cifuentes, M., J. Jobse, V. Watson, y B. Kauffman. 2004. Determinación de Carbono en Suelos de Diferentes Tipos de Uso de Tierra a lo Largo de una Gradiente Climática en Costa Rica. Portland (EEUU): USDA.

Díaz-Gustavo, N., M. Martínez-Salvador, J. L. García- Hernández, M. Norzagaray-Campos, A. Luna- González, H. A. González-Ocampo. 2015. "Carbon Sequestration of Caesalpinia platyloba S. Watt (Leguminosae) (Lott 1985) in the Tropical Deciduous Forest." PLoS ONE $10 \quad$ ( 5 ): $\quad$ e 0125478 . doi:10.1371/journal.pone.0125478

Douterlungne, D., A. M. Herrera Gorocica, B. G. Ferguson, I. Siddique, y L. Soto Pinto. 2013. "Ecuaciones Alométricas Para Estimar Biomasa Y Carbono De Cuatro Especies Leñosas Neotropicales Con Potencial Para La Restauración.” Agrociencia 47 (4): 385-397.

MINAGRI. 2010. “Decreto Supremo Nº13- 2010AG.”Lima(Perú): MINAGRI

FAO. 2002. Captura de carbono en los suelos para un mejor manejo de la tierra. Informes sobre recursos mundiales de suelo. Roma (Italia): FAO.

García, Y., W. Ramírez, S. Sánchez. 2012. “Indicadores De Calidad De Los Suelos: Una Nueva Manera De Evaluar Este Recurso.” Pastos y Forrajes 35 (2): 125-138.

Gorbitz, G. 2001. Determinación de las Reservas de Carbono en la Biomasa Aérea en Plantaciones de 8 años de Calycophyllum spruceanum b. en el Valle del Aguaytía. Tesis de Grado. Universidad Nacional Agraria La Molina. Lima (Perú).

González, M. 2008. Estimación de la Biomasa Aérea y La Captura De Carbono En Regeneración Natural De Pinus maximinoi H. E. Moore, Pinus oocarpa var. ochoterenai Mtz. y Quercus sp. en el Norte del Estado de Chiapas, México. Tesis de Maestría. CATIE. Turrialba (Costa Rica).

IPCC. 2001. El Cambio Climático. Ginebra (Suiza): IPCC

Kanowski, J., y C. P. Catterall. 2010. “Carbon Stocks in Above-Ground Biomass of Monoculture Plantations, Mixed Species Plantations and Environmental Restoration Plantings in North-East Australia." Ecological Management \& Restoration. 11 (2): 119-126.

Luza, I. 2015. "Stock de Carbono Almacenado en la Biomasa Aérea, Sotobosque y Suelo en Bosques Andinos, Huancayo, Perú, 2013." RevistaECIPerú 11 (2): 33-41.

Martínez E., J. P. Fuentes, y E. Acevedo. 2008. “Carbono Orgánico y Propiedades del Suelo." Revista de la Ciencia del Suelo y Nutricon Vegetal 8 (1): 68-96.

Pérez, M. E., A. López, M. Asturías, J. López, E. Zuñiga, W. Velásquez, B. Monroy, R. Méndez, T. Calderón, M. Castillo, G. Hernández, C. Franco, D. Roldan, S. Sánce, J. Marcos, y S. Velásquez. 2015. "Los Árboles Como Reguladores de Temperatura y Almacenaje de Carbono: Estudio Preliminar Sobre los Servicios Eco- 
sistémicos y Percepción Social en el Municipio de Chiquimula." Ciencia \& Conservación 6(1):25-40.

Pérez, R. J., y S. M. Oliva. 2013. Determinación de Carbono en la Biomasa Aérea de Sistema Agroforestal de Producción Coffe arabica "Café", en Asociación con Myrsine oligophylla "Morocho", Caesalpnia spinosa "Tara", Inga edulis "Guaba" y Acacia macracantha "Huarango" en la Cuenca Media del Río Utcubamba. Amazonas. Chachapoyas (Perú): INDES-CES.

Picard N., L. Saint-André, y M. Henry. 2012. Manual de Construcción de Ecuaciones Alométricas Para Estimar el Volumen y la Biomasa de los Árboles: Del Trabajo de Campo a la Predicción. Rome (Italia): FAO y CIRAD.

Quispe, K., M. Mamani, J. I. Rodríguez, S. Marcas, M. Romero, D. Meza, J. Quispecuro, J. Soto, y D. Rodrigo. 2012. Simulación de la remoción de carbono en la especie: Caesalpinia spinosa (Tara). Lima (Perú)

Romanya J., P. Rovira, y R. Vallejo. 2007. “Análisis del Carbono en los Suelos Agrícolas de España. Aspectos Relevantes en Relación a la Conservación a la Agricultura Ecológica en el Ámbito Mediterráneo." Ecosistemas 16 (1): 50-57.

Valenzuela, H. 2001. Estimación de Secuestro de Carbono en Bosques Naturales de Oyamel (Abies religiosa) en el Sur del Distrito Federal. Tesis de Grado. Universidad Autónoma Chapingo. Chapingo (México).

Vela, G., J. López, y M. L. Rodríguez. 2011. "Niveles de Carbono Orgánico Total en el Suelo de Conservación del Distrito Federal, Centro de México." Investigaciones Geográficas (77): 18-30.

Zambrano, A., F. Franquis, y A. Infante. 2004. "Emisión y Captura de Carbono en los Suelos en Ecosistemas Forestales." Revista Forestal Latinoamericana 19(35): 11-20. 